\title{
Re: The relationship between head injury and facial trauma
}

\author{
Amit Agrawal
}

Received: 18 August 2013 / Accepted: 11 November 2013 / Published online: 26 November 2013

(C) Springer-Verlag Berlin Heidelberg 2013

\section{Dear Sir,}

We read the article "The relationship between head injury and facial trauma: a case-control study" [1] with great interest. In the present prospective study, the authors discuss their experience with the pattern of facial bone fractures and their correlation with intracranial injuries. The patients were categorized into concomitant maxillofacial and head injuries (the "study group") and patients with maxillofacial trauma without associated head injuries (the "control group"). [1] In the workup of the patients, the details of facial trauma, including medical history, physical examination, radiographic evaluation, and many other consultations (details not available here) were included. [1] The definition of the head injury is not clear as the patients with skull fracture and intracranial injuries were only included. It is not clear how many patients with any systemic injuries were included or excluded. In the section about the causes of injury, particularly the violence in itself, does not clarify the severity of impact as it can range from domestic violence to violent street agitations; also, sports-related injuries can range from a football match to motor racing. In the present study, the intracranial injuries were categorized as concussion, cerebral contusion, and intracranial hemorrhages; however, there were no clear-cut criteria whether it was a clinical diagnosis or imaging findings (either $\mathrm{x}$-ray or CT scan). It is not clear that the patient who had head injury (means 302 patients) only underwent neurosurgical evaluation and $\mathrm{CT}$ scan as there are no details regarding how many patients were investigated for the intracranial injuries and how was the head injury ruled out in other patients (although the definition of the head injury in itself is not clear). It is not clear whether the patients in the control group underwent CT scan; if yes, then how many patients had abnormal imaging in non-head injury (control) group? And if no, then how was head injury ruled out? It is also not clear how the diagnosis of facial injuries was made or confirmed (facial x-rays of CT scan). Although in conclusion, it has been suggested that "the patients with concomitant head and facial injuries had a high GCS score (12-15) at the time of admission" [1] However, it has not been well supported by the results of the study.

\section{Reference}

1. Zandi M, Seyed Hoseini SR (2013) The relationship between head injury and facial trauma: a case-control study. Oral and maxillofacial surgery 17:201-207

\footnotetext{
A. Agrawal ( $\square)$

Narayana Medical College and Hospital, Chintareddy Palem,

Nellore, Andhara Pradesh, India

e-mail: dramitagrawal@gmail.com
} 